\title{
17. Analysis of Selected Hominoid Joint Surfaces Using Laser Scanning and Geometric Morphometrics: A Preliminary Report
}

William E. H. Harcourt-Smith*

Department of Vertebrate Paleontology

American Museum of Natural History

Central Park West \& 79th Street

New York, NY 10024, USA

willhs@amnh.org

Melissa Tallman

Anthropology PhD Program

CUNY Graduate School

New York, NY 10016, USA

\& NYCEP

tallman@nycep.org

Stephen R. Frost

Department of Anthropology

University of Oregon

Eugene, OR 97403, USA

sfrost@uoregon.edu

David F. Wiley

Institute for Data Analysis and Visualization

University of California

Davis, CA 95616, USA

wiley@cs.ucdavis.edu

F. James Rohlf

Department of Ecology and Evolution

Stony Brook University

Stony Brook, NY 11794, USA

NYCEP

rohlf@life.bio.sunysb.edu

Eric Delson

Department of Anthropology

Lehman College/CUNY

\& Department of Vertebrate Paleontology

American Museum of Natural History

Central Park West \& 79th Street

New York, NY 10024, USA

\& NYCEP

eric.delson@lehman.cuny.edu

* Address for correspondence: willhs@amnh.org

(C) Springer Science + Business Media B.V. 2008 


\subsection{Foreword}

Fred Szalay is a polymath of evolutionary morphology. From the mid-1960s (Szalay, 1968) to at least the mid-1980s (Szalay et al., 1987), he was acknowledged as the leading researcher on non-anthropoid fossil primates, complementing the anthropoid expertise of Elwyn Simons who had just laid the foundations for paleoprimatology as a distinct field through his revisions and field success in the Fayum of Egypt. Fred edited two volumes in this area in 1975, in both of which Delson (as a very junior colleague) was most pleased to be included (Szalay, 1975; Luckett and Szalay, 1975), which in turn led to their collaborative review of the whole order (Szalay and Delson, 1979). Fred was also an early critic of the cladistic approach to phylogenetic reconstruction, passionately opposed to the narrowness of evolutionary thinking that he felt stemmed from cladistic thinking, so he developed (often with Walter Bock) a refinement of the "evolutionary" taxonomic approach (e.g., Szalay, 1977b, 1993; Szalay and Bock, 1991). Although his earliest work focused on crania and dentitions, Fred was equally interested in postcranial morphology from both functional and phylogenetic viewpoints (Decker and Szalay, 1974; Szalay and Decker, 1974; Szalay et al., 1975); he almost single-handedly made morphology of the postcranium relevant to mammalian phylogeny reconstruction, and he argued that the distinction between functionalism and non-functional thinking was entirely artificial. His long 1977(a) paper on mammalian phylogeny was almost entirely based on the evidence from foot bones, and this focus continued in his work on primates (Szalay and Dagosto, 1980, 1988; Szalay and Langdon, 1986) as well as eutherian and especially metatherian mammals (Szalay and Drawhorn, 1980; Szalay, 1984, 1994; Szalay and Lucas, 1993, 1996; Szalay and Sargis, 2001). Fred argued that taxa could just as readily be distinguished by their postcrania as by their teeth, ear regions or facial structure. Many of his colleagues rejected this idea or thought that (at best), postcranial morphology might delineate families or subfamilies, but not genera or species. Fred's most recent contribution (Szalay, 2007; in a book whose co-editor is the same as this volume's) reviewed the locomotor adaptations of the earliest primates and their predecessors, comparing several entrenched hypotheses unfavorably to his own prior interpretations.

We hope Fred will appreciate this paper, which attempts to distinguish genera and even individuals from the morphology of their ankles, one of his favorite anatomical regions. On the other hand, we realize that Fred never cared much for complex statistical analyses (though he adopted a variety of relatively high-tech approaches after their usefulness was demonstrated to him), and we hope he will not be too put off by this work on that basis.

\subsection{Introduction}

Allocating fossil specimens to a particular taxon, and even in some cases to a particular individual, is a primary problem in paleontology. For many mammals, including primates, fossil taxa are defined from craniodental morphology, and postcranial elements can often be allocated only when directly associated with cranial parts. Moreover, when multiple individuals are recovered, it is usually important to associate elements of a single individual in order to help determine functional adaptations, overall size and proportions, as well as other factors, such as the number of individuals preserved.

In addition to careful taphonomic analysis, most studies attempting to allocate isolated postcranial elements of unknown association, particularly of fossil hominins, have used a combination of visual estimation of morphological similarity and linear measurements (whether analyzed in a uni-, bi-, or multivariate manner). Central to this study is the concept of joint congruence, that is, the closeness of fit between the articulating elements of a joint complex. Two previous publications (Aiello et al., 1998; Wood et al., 1998) reported the results of preliminary analyses using a laser surface scanner (LSS) to compare congruence in the talo-crural joint in hominoids and one human fossil. Their work represents an important new research direction, but they were unable to continue along this line because the statistical techniques, the computer software and hardware, and the models they developed, were unable to address the complex 3D relationships between the reciprocal shapes involved.

It is clear that the effect of soft tissue (cartilage, ligament, tendon and musculature) is an important part of congruence in most joints (see, e.g., Hamrick, 1999). However, fossil and archaeological material does not usually preserve soft tissue structures, and researchers nearly always have to work with hard tissue joint surfaces preserved as subcondral bone. As we are ultimately interested in matching previously unassociated fossil and archaeological elements, we considered it desirable to use the hard-tissue joint surfaces of extant specimens housed in museum collections. In that context, we present preliminary results from a new approach to joint congruence. This new approach to matching is an indirect one that only requires a strong covariation between the shapes of the matching pairs of structures. We combine laser surface scans of opposing joint surfaces with geometric morphometrics and multivariate statistical analyses to examine ways to differentiate taxa and match elements from the same individual, utilizing an initial sample of extant hominoids. We use the tibial and talar components of the ankle joint because it is a relatively "tight" and predominantly uniaxial joint. As a result, there is a reasonable expectation that the reciprocal surface should be relatively congruent.

\subsection{Background}

There have been a number of studies on individual joint articular surfaces, as well as reciprocal joint surface geometry and congruence of such complexes as the gleno-humeral, humeroulnar, radio-ulnar, carpo-metacarpal, metacarpo-phalangeal, femoro-tibial, patello-femoral, and tarso-metatarsal joints. These studies have mainly focused on documenting the normal anatomy of the skeletal elements involved in each joint 
complex (Leardini et al., 1999; Matsuda et al., 1998; Medley et al., 1983; Rostlund et al., 1989; Shiba et al., 1988; Siu et al., 1996; Soslowsky et al., 1992; Staron et al., 1994; Tamai et al., 1988; Yoshioka et al., 1988).

Many of the biomedical studies have dealt with joint geometry and mechanics and how this information is utilized in prosthesis design (Hertel and Lehmann, 2001; Hertel et al., 2002; Leardini, 2001; Roberts et al., 1991; Swieszkowski et al., 2001). Knowledge of normal and pathological joint morphology and kinematics allows for the design and implantation of prostheses that accurately approximate normal range and quality of motion (Bullough, 1981; Frost, 1999; Haut et al., 1998; Hlavacek and Vokoun, 1998; Kauer and de Lange, 1987; Kelkar et al., 2001; Pretterklieber, 1999; Soslowsky et al., 1992; Waide et al., 2000). Anthropological studies include those that have examined patterns of joint size dimorphism in the elbow and knee of catarrhine primates (Lague, 2003), patterns of sexual dimorphism in hominoid humeri (Lague and Jungers, 1999), patellar articular proportions of recent and Pleistocene humans (Trinkaus, 2000), and the relationship between hip joint congruence and function (MacLatchy and Bossert, 1996; MacLatchy, 1996).

Various methodologies have been employed in these studies to understand (and in some cases to visualize) joint geometry and morphology. Methods utilized include: digitization of joint facets (Dykyj et al., 2001; Yoshioka et al., 1988); Magnetic Resonance imaging (Matsuda et al., 1998; Staron et al., 1994; Staubli et al., 1999); Merchants's Skyline views \& Axial Computed Arthro-tomography (CTA) (Walker et al., 1993); Stereophotogrammetry (SPG) (Ateshian et al., 1992; Huiskes et al., 1985; Kelkar et al., 2001; Soslowsky et al., 1992); three-dimensional Coordinate Digitizing System (3DCDS) (Haut et al., 1998); computed tomography (CT) (Siu et al., 1996); X-ray absorptiometry scans (Mikhail et al., 1996); and classical morphometrics (e.g., Rostlund et al., 1989). Most recently, Tocheri et al. (2003) have studied modern and fossil trapezia with the aid of LSS data, but their methodology was not designed to take full advantage of those data in terms of estimating joint congruence.

\subsection{Materials}

The sample consists of the left tibia and talus of 22 extant Homo sapiens, 20 Pan troglodytes and 12 Gorilla gorilla. All individuals are adults and numbers of males and females are as even as was possible to arrange. The modern human sample includes individuals representing Alaskan Inuit from Point Hope $(n=7)$, ancient Egyptians $(n=3)$, archaeological Native Americans from Canyon Del Muerto, Arizona (n $=5)$ and modern African- $(\mathrm{n}=5)$ and Euro-Americans ( $\mathrm{n}$ = 2) from New York medical school collections, studied by courtesy of the Department of Anthropology, American Museum of Natural History, New York; the Pan and Gorilla samples are composed of wild-shot adults housed in collec- tions within the museum's departments of Anthropology and Mammalogy.

\subsection{Methods}

Whole tibiae and tali were scanned with either a Cyberware 3030 laser surface scanner (Cyberware Inc., 2110 Del Monte Av., Monterey, CA 93940) or a portable Minolta Vivid 910 laser surface scanner (Konica Minolta Photo Imaging USA Inc., P.O. Box 92253, Chicago, IL 60675). The Cyberware 3030 is capable of scanning to a resolution of $\sim 300$ microns in the $\mathrm{z}$ plane, and the Minolta Vivid 910 scanner to $\sim 30$ microns. Resulting data files were edited and processed using CyDir (Cyberware Inc.) or Geomagic Studio 8.0 (Geomagic Inc., 3200 Chapel Hill-Nelson Rd., Research Triangle Park, NC 27709) respectively. The output files were saved in the .ply polygon model file format capable of storing additional information such as color and surface normals, thus providing highly accurate surface renditions of the actual object scanned, in this case whole bones. The .ply files were then entered into Landmark Editor (Wiley, 2006), a software package written for our team's LSS research, where the user can visualise the images as NURBS (Nonuniform rational B spline) surfaces.

A grid of points was then placed on the "virtual" joint surface of each laser scan. This grid was anchored by eight discrete homologous landmarks placed at identifiable features along the perimeter of the articular surface, and one in the center, of the distal tibia and talar trochlea. These landmarks were adapted from talar landmarks devised by HarcourtSmith (2002) and tibial landmarks devised by Harcourt-Smith et al. (2004) and Garcia and Harcourt-Smith (2006). Table 17.1 summarizes the definitions for positioning these landmarks. Levels of landmark homology were determined after Bookstein (1991) and O'Higgins (2000).

The grid-defined articular areas were then re-sampled into a three-dimensional mesh of 361 ( 19 x 19) evenly-spaced points. Using an odd number of semi-landmark points makes it possible to correctly place a middle line of points along a linear anatomical structure, in this case the trochlear groove of the talus. Moreover, it was determined by visual inspection that the $19 \times 19$ grid created a very dense distribution of points on structures as small as the distal tibial articular surface and talar trochlea. In that respect, this number of points was deemed sufficient to capture the shape of the articular surfaces.

Landmark Editor automatically spaces these points by calculating the distance between two landmarks along the surface of the polygon model and then evenly spacing points along that line according to a number specified by the user. This methodology can be applied to either a line or a grid of points. These points are called semilandmarks because their relative location on the surface is arbitrary, and only their variation in directions orthogonal to the surface reflect differences in the shape of the surface (see Bookstein, 1997; Delson et al., 2001; Gunz et al., 2005; 
TABLE 17.1 Homologous landmarks taken on the talar trochlear surface and distal tibial articular surface. Talar landmarks are after HarcourtSmith (2002) and tibial landmarks adapted from Harcourt-Smith et al. (2004) and Garcia and Harcourt-Smith (2006). The types of landmark (i.e., level of homology) follow Bookstein (1991) and O’Higgins (2000).

\begin{tabular}{|c|c|c|}
\hline \multicolumn{3}{|c|}{ Talar Landmarks } \\
\hline Number & Type & Description \\
\hline 1 & III & Most distal point of the trochlear groove. \\
\hline 2 & II & Most distal point of contact between the medial malleolar facet and the trochlear surface. \\
\hline 3 & III & Most dorsal point on the medial facet margin. \\
\hline 4 & II & Most proximal point of contact between the medial malleolar facet and the trochlear surface. \\
\hline 5 & III & Most proximal point of the trochlear groove. \\
\hline 6 & II & Most proximal point of contact between the lateral malleolar facet and the trochlear surface. \\
\hline 7 & III & Most dorsal point on the lateral facet margin. \\
\hline 8 & II & Most distal point of contact between the lateral malleolar facet and the trochlear surface. \\
\hline 9 & III & Most dorsal point on the trochlear groove. \\
\hline \multicolumn{3}{|c|}{ Tibial Landmarks } \\
\hline Number & Type & Description \\
\hline 1 & II & Point where anterior and lateral facet margins meet. \\
\hline 2 & III & Midpoint between landmarks 1 and 3 along the lateral side of the articular surface. \\
\hline 3 & II & Point where posterior and lateral facet margins meet. \\
\hline 4 & III & Midpoint between landmarks 3 and 5 on the posterior facet margin. \\
\hline 5 & II & $\begin{array}{l}\text { Point where posterior and medial facet margins meet. Point should be just before surface rises } \\
\text { to become the medial malleolus. }\end{array}$ \\
\hline 6 & III & $\begin{array}{l}\text { Midpoint on the medial side of the articular surface between landmarks } 5 \text { and } 7 \text {. } \\
\text { Landmark should be on the edge just before it rises for the medial malleolus. }\end{array}$ \\
\hline 7 & II & $\begin{array}{l}\text { Point where medial and aneterior facet margins meet. Point should be just before it } \\
\text { rises to become the medial malleolus. }\end{array}$ \\
\hline 8 & III & Midpoint between landmarks 9 and 1 on the anterior facet margin. \\
\hline 9 & III & Middle of articular surface. \\
\hline
\end{tabular}

and Figure 17.1). The same number of semilandmarks was used on all specimens.

The surfaces, as characterized by the sets of landmarks and semilandmark points, were then registered using Generalized Procrustes Analysis (GPA), which removes the effects of variation in orientation, location, and size (Rohlf and Slice, 1990). In addition, the effects of variation due to the somewhat arbitrary spacing in different specimens of the semilandmarks over the sampled surface were minimized using a "sliding" process. During registration, the semilandmarks are slid along planes tangent to the sampled surface around each semilandmark so as to minimize the Procrustes distance among configurations (Figure 17.2), so that the effects of the arbitrary positioning of semilandmarks on the surface are mitigated. This technique, originally applied to 2D curves (Bookstein, 1997), has recently been extended to surfaces (Gunz et al., 2005) using bending energy as the sliding criterion. In this study, however, the criterion being minimized was Procrustes distance rather than bending energy (Rohlf, 2005). Results obtained with bending energy or Procrustes distance as the minimized criterion were recently shown to be comparable (Perez et al., 2006). The sliding algorithm did not work well for the points on the grids of semilandmarks representing the malleolar facets of the tibia and talus, as it caused considerable erroneous deviation of a number of semilandmarks. This is being further investigated, but as a result only analyses of the trochlear surfaces of the talus and tibia are presented. Both GPA and sliding were completed using code written by F.J.R

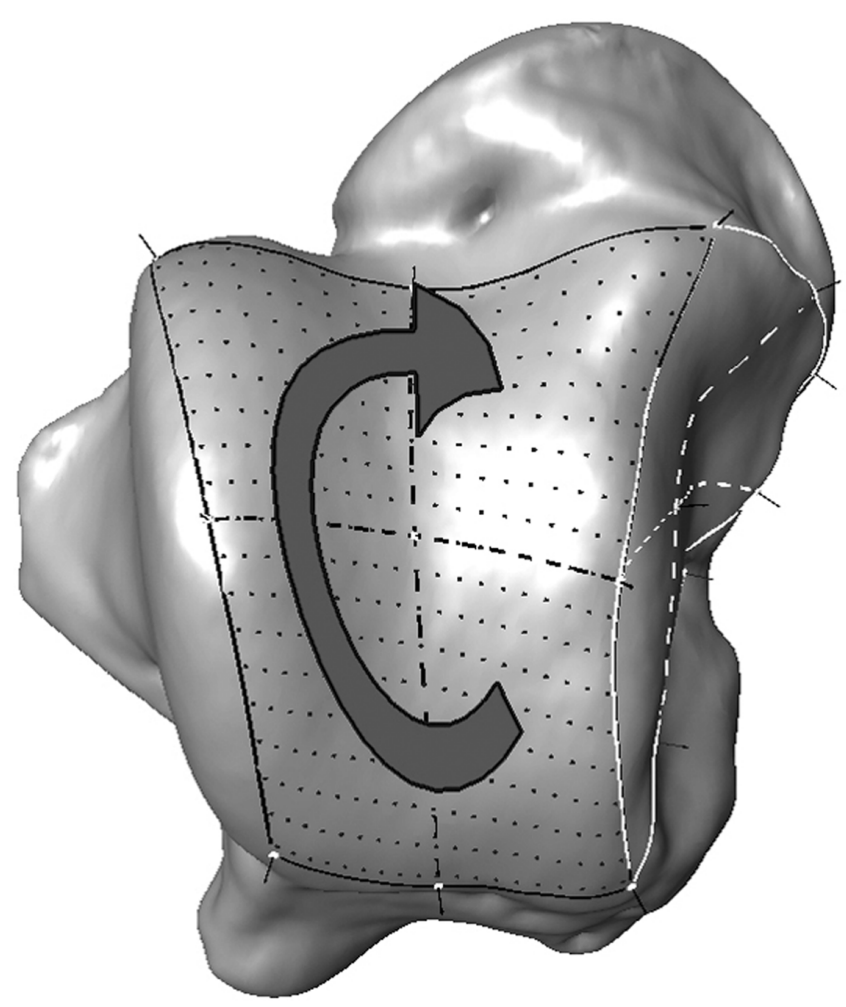

FIGURE 17.1. Screen capture from Landmark Editor software, showing the dorsal view of a laser scan of a modern human talus. On the trochlear surface is the dense $19 \times 19$ grid of semilandmark points. The arrow represents the order in which the points are collected. 

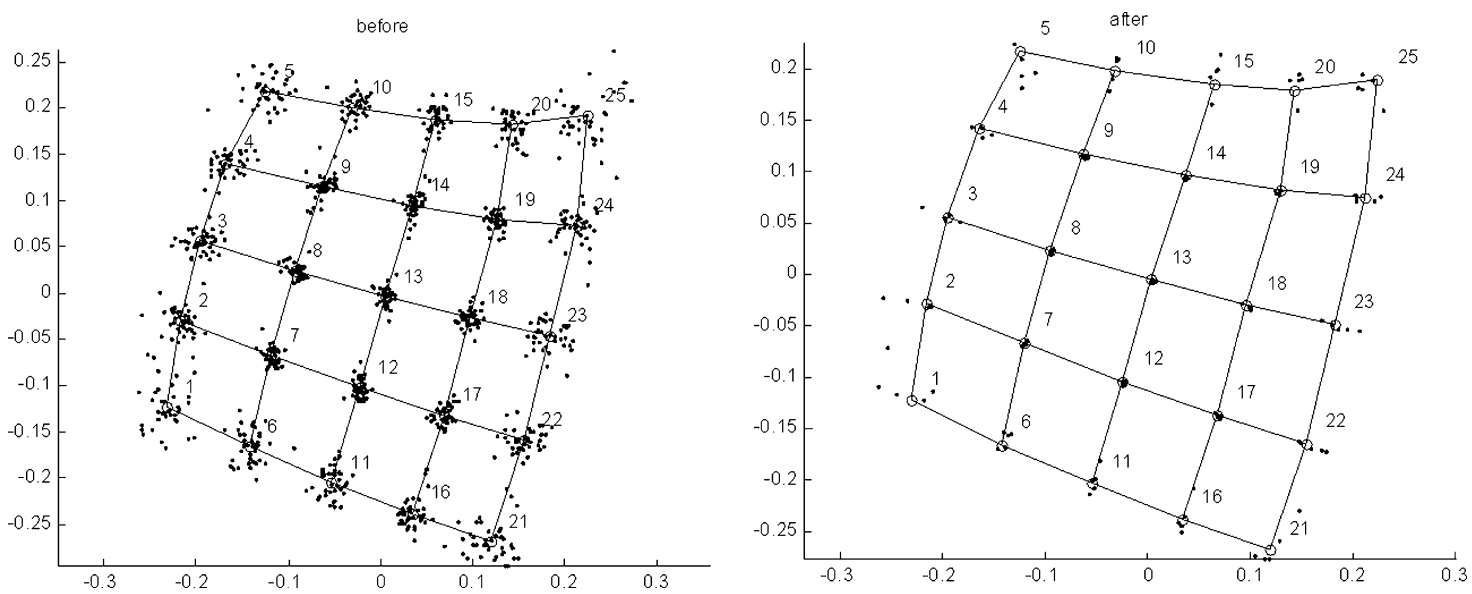

FIGURE 17.2. Unslid (left) versus slid (right) grids of semilandmarks. In this example, only $5 \times 5$ landmark grids are shown for ease of visualization. Black dots represent semilandmarks, the grid is for visualization only. Sliding was achieved using Procrustes distance as the criterion. Note positioning of semilandmarks on the surface is equalized, but their elevation normal to the surface is preserved.

for MATLAB software (The Mathworks, Inc., Natick, MA). After GPA and sliding, size was restored to the data (i.e., backscaled) by multiplying each coordinate by centroid size using SAS (SAS Institute, Cary, NC).

\subsection{Data Analysis}

All data analysis was conducted using SAS. Two Block Partial Least Squares (2B-PLS) or Singular Warps Analysis was used to analyze the relationships between the morphologies of the opposing joint surfaces. This technique analyzes the covariation between two sets of variables (Rohlf and Corti, 2000; Bookstein et al., 2003; Frost et al., 2003). In this study, these are the shape variables (i.e., the 3D coordinates of landmarks and semilandmarks) of the opposing joint surfaces, with the tibia comprising one matrix and the talus the other. This is a novel application of the technique, using the information on covariation between the shapes of opposing surfaces to detect whether a pair of structures has in fact been correctly associated.

This technique has a potential advantage over alternative approaches that rely on directly aligning the reciprocal joint surfaces to evaluate their congruence (e.g., assessing the volume separating "optimally" positioned surfaces). Such approaches require assumptions as to the distribution of the thickness of joint cartilage as well as the habitual posture and range of motion of those joints in the organisms involved (i.e., assumptions are made about how the opposing joint surfaces should articulate). None of these are required here. Instead, this approach simply assumes that a relationship exists between the shapes of opposing joint surfaces (i.e., they covary), and that the shapes of mismatched joint surfaces deviate from this baseline. More specifically, a matrix of covariances between shape variables based on correctly paired structures is decomposed using a singular-value decomposition to yield linear combinations of variables from each of the structures that best account for the observed covariation between the paired structures (Rohlf and Corti, 2000; Bookstein et al., 2003). Pairs of scores are then computed for the correctly paired structures. The scores for the first singular vector from each structure are expected to show a tight pattern of correlation. Pairs of scores are also computed for the incorrectly matched structures using the matrices of singular vectors based on the matched data. Scores for mismatched specimens are expected to fall outside of the pattern of covariation for correctly matched surfaces. That is, the fit of a particular pair of joint surfaces is compared to the pattern of covariation of matched pairs. As noted, no assumptions are made about how the opposing joint surfaces should articulate (as one might do by manually fitting them together).

Finally, the PLS scores of the first ten singular warps were subjected to a canonical discriminant function (DF) analysis, also called a canonical variates analysis (see Hubberty, 1994) to explore whether pairs of reciprocal joint surfaces could be correctly classified as matched or mismatched with any degree of certainty. To compute the reclassification rates, a cross validation procedure was used, where each pair was removed in turn from the data, the discriminant functions recomputed from the remaining pairs, and then that pair reclassified. All analyses were conducted on landmark data that were subjected to sliding and backscaled using centroid size as a multiplier, because the results from the DF analysis were considerably better using sliding and backscaled data than without.

\subsection{Results}

Sliding the semilandmarks gives considerably better results than not sliding them. Specifically, where the data were slid, but there was no backscaling, $84 \%$ of pairs were correctly classified. For unslid data, where backscaling (using centroid 
size as a multiplier) was included, the rate of correct classification rose to $92 \%$. Finally, the backscaled and slid data produced the highest rate of correct classification, at $95 \%$. Therefore, only the results of analyses performed after sliding are shown here. For the full dataset, PLS of the slid and unslid semilandmark grids does not produce any significant differences between matched and unmatched individuals when size is adjusted for using GPA. However, results from the backscaled data were more positive. Figure 17.3 shows a bivariate plot of PLS axis 1 for both the tibia (x-axis) and the talus (y-axis) using the backscaled data. The matched specimens form a relatively narrow diagonal "band" running from the intersect of the $\mathrm{x}$ and $\mathrm{y}$ axes at roughly a $45^{\circ}$ angle. The intergenerically mismatched specimens predominantly fall away from this line, either above or below it; in each case, the name of the genus providing the tibia precedes that yielding the talus. The Pan-Homo and Pan-Gorilla pairs fall exclusively above the line of matched specimens. For the Homo-Pan and Homo-Gorilla pairs, most specimens fall away from the band of matched individuals, but a number of these mismatches do fall within the range of matches. The Gorilla-Pan and Gorilla-Homo mismatches also fall partially within the range of matched individuals, and partially outside it. Overall, $76 \%$ of intergeneric mismatches fell outside the range of variation of the matched individuals.

In the above analysis, matched and mismatched humans did not significantly differ. However, when the analysis was restricted to Homo alone (Figure 17.4), there was a strong separation between pairs from the same or different indi- viduals: $73 \%$ of modern human mismatched individuals fell outside the range of variation of matched specimens, either above or below the latter's diagonal band.

Finally, canonical discriminant function (DF) analysis was conducted on the first 10 PLS scores for the tibia and talus where size had been restored as above, using centroid size as a multiplier. Table 17.2 summarizes the rates of correct allocations for each set of matched and unmatched pairings. For the intrageneric pairs, $100 \%$ of Gorilla, 95\% of Pan and $93.75 \%$ of Homo were correctly re-allocated to genus. For the intergeneric mismatched pairings, $90.9 \%$ of Gorilla-Pan (i.e., Gorilla tibia and Pan talus) mismatches were correctly assigned to that group, while $100 \%$ of the Pan-Gorilla pairs were correctly assigned. All of the 3 Homo -Gorilla mismatches were correctly assigned, 8 of the 9 Homo -Pan mismatches, and all of the 12 Pan-Homo pairings. Overall the percentage of correct assignments was high for all combinations of matched and unmatched individuals.

Figure 17.5 shows the canonical scores for DF 1 (x-axis) versus DF 2. Most of the pre-assigned groups form tight, distinct clusters separated from each other. Both the Homo and Pan intrageneric groups have very little or no overlap with any other group. DF 1 separates the intra-Homo group and the PanHomo group (on the negative end of the axis) from all others. There is also some separation between the Homo-Pan, GorillaPan and Pan groups and the Pan-Gorilla, Homo-Gorilla and Gorilla groups. The relative position of any group on DF1 seems to be predominantly influenced by the second taxon of the pair, i.e., that represented by talar landmarks, with Homo at

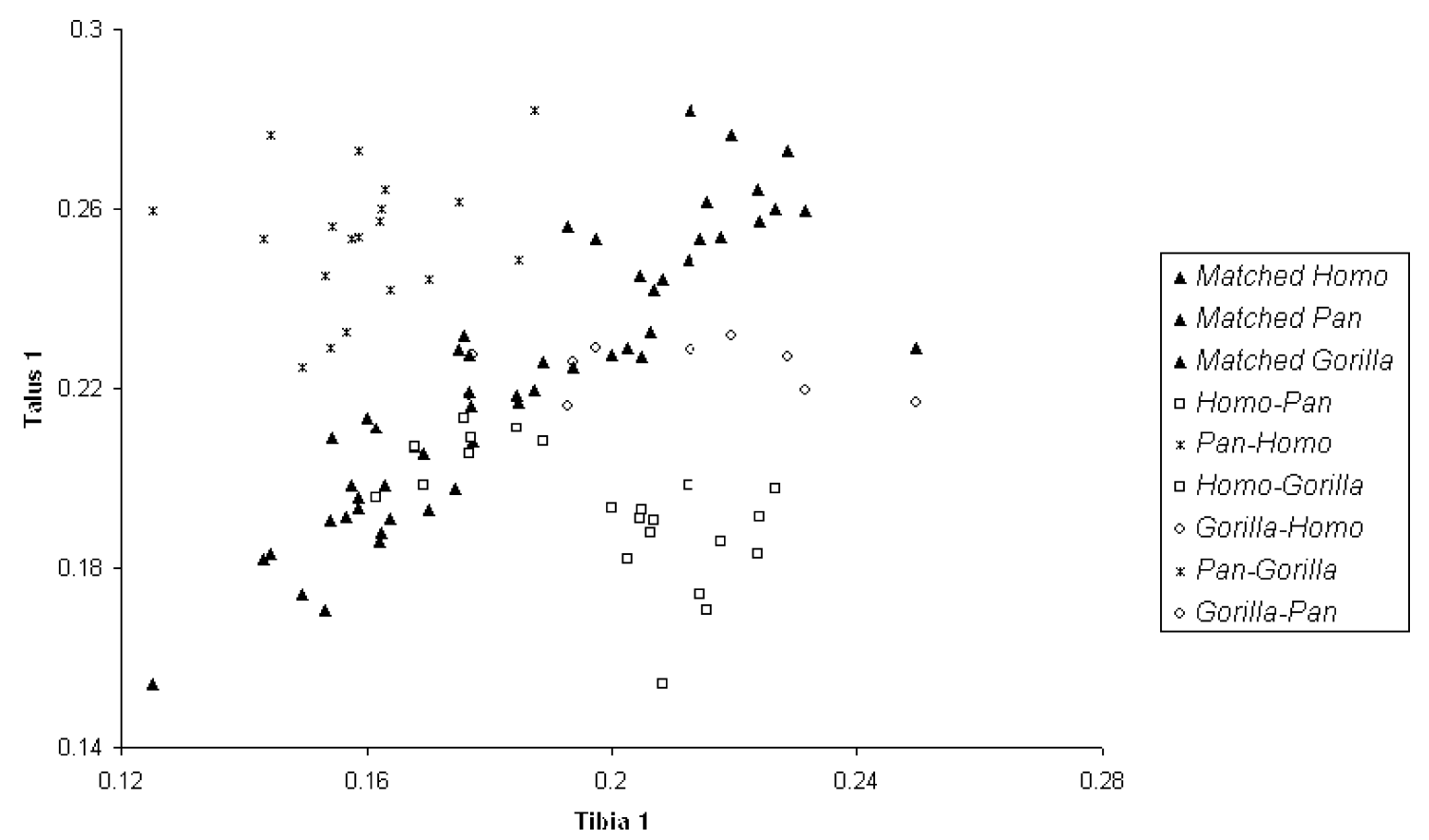

FIGURE 17.3. PLS (singular) vector 1 scores (after sliding and backscaling) for tibio-talar pairs; tibia on the x-axis and talus on the y-axis. All intraspecific pairs, whether from the same or different individuals, are given the same labels (solid triangles) for visual clarity. For each interspecific pairing, the first taxon in the legend refers to the tibial surface, the second to the talar. 


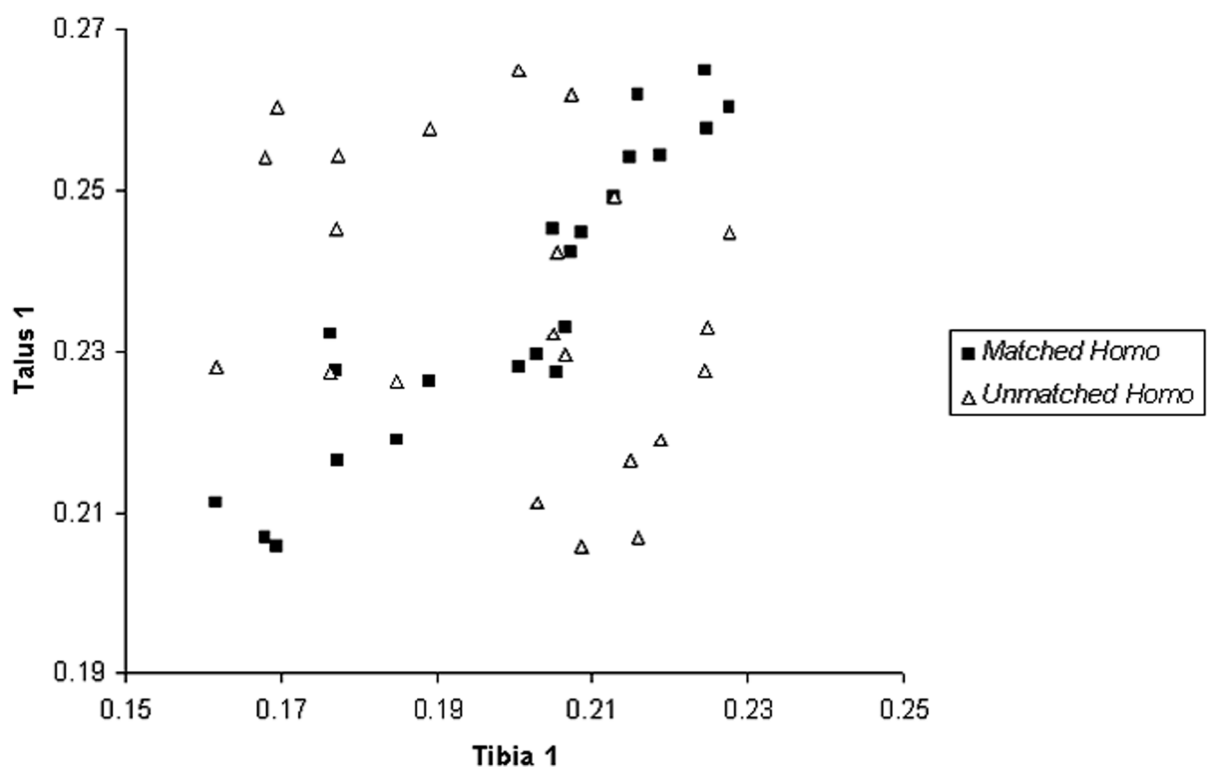

FIGURE 17.4. PLS (singular) vector 1 scores (after sliding and backscaling) for tibio-talar pairs; tibia on the x-axis and talus on the y-axis. Results for modern humans only, pairs from same individuals (matched) vs. random combinations.

TABLE 17.2. Frequency of correct and incorrect allocations from discriminant function analysis of PLS scores. For this DF analysis, a cross validation procedure was used. The actual pairing is shown on the left, the categories into which pairs were classified are listed across the top. For each pairwise comparison, the upper value is the number of allocations for that category, and the lower value (bold) is the corresponding percentage. Values along the major axis report correct reclassifications, while off-axis values are misclassifications where, for example, a Gorilla tibia and Pan talus was reported as Pan + Pan. $\mathrm{H}=$ Homo, $\mathrm{G}=$ Gorilla, $\mathrm{P}=$ Pan. First letter of a pair represents the taxon of the tibial surface, and the second the talar.

\begin{tabular}{|c|c|c|c|c|c|c|c|c|c|}
\hline \multicolumn{10}{|c|}{ Classified as } \\
\hline & $G+G$ & $G+P$ & $\mathrm{H}+\mathrm{G}$ & $\mathrm{H}+\mathrm{H}$ & $\mathrm{H}+\mathrm{P}$ & $P+G$ & $\mathrm{P}+\mathrm{H}$ & $\mathrm{P}+\mathrm{P}$ & Total \\
\hline Actual & 11 & & & & & & & & 11 \\
\hline$G+G$ & $100 \%$ & & & & & & & & \\
\hline & & 10 & & & & & & 1 & 11 \\
\hline$G+P$ & & $90.9 \%$ & & & & & & $9.1 \%$ & \\
\hline & & & 3 & & & & & & 3 \\
\hline $\mathrm{H}+\mathrm{G}$ & & & $100 \%$ & & & & & & \\
\hline & & & & 30 & & & 2 & & 32 \\
\hline $\mathrm{H}+\mathrm{H}$ & & & & $93.75 \%$ & & & $6.25 \%$ & & \\
\hline & & & & & 8 & & & 1 & 9 \\
\hline $\mathrm{H}+\mathrm{P}$ & & & & & $88.9 \%$ & & & $11.1 \%$ & \\
\hline & & & & & & 8 & & & 8 \\
\hline$P+G$ & & & & & & $100 \%$ & & & \\
\hline & & & & & & & 12 & & 12 \\
\hline $\mathrm{P}+\mathrm{H}$ & & & & & & & $100 \%$ & & \\
\hline & & & & & 1 & & & 19 & 20 \\
\hline $\mathrm{P}+\mathrm{P}$ & & & & & $5 \%$ & & & $95 \%$ & \\
\hline & & & & & & & & & 101 \\
\hline Total correct & & & & & & & & & $95.3 \%$ \\
\hline & & & & & & & & & 5 \\
\hline Total incorrect & & & & & & & & & $4.7 \%$ \\
\hline & & & & & & & & & 106 \\
\hline Grand total & & & & & & & & & $100 \%$ \\
\hline
\end{tabular}

the negative end of the axis, Pan intermediate, and Gorilla at the positive extreme. It is interesting to note that these are not arranged according to size, as the Pan talar and tibial surfaces are smaller than those of Homo. On DF2, the principal separation is between the Homo-Pan, Gorilla-Pan and Pan groups and all others. The intrageneric groups all fall closer to the middle of DF2, suggesting that it is intergeneric mismatches that are mainly driving variation on this axis. In other words, the intergeneric mismatches essentially fall outside the range of variation seen within the intrageneric matches. 


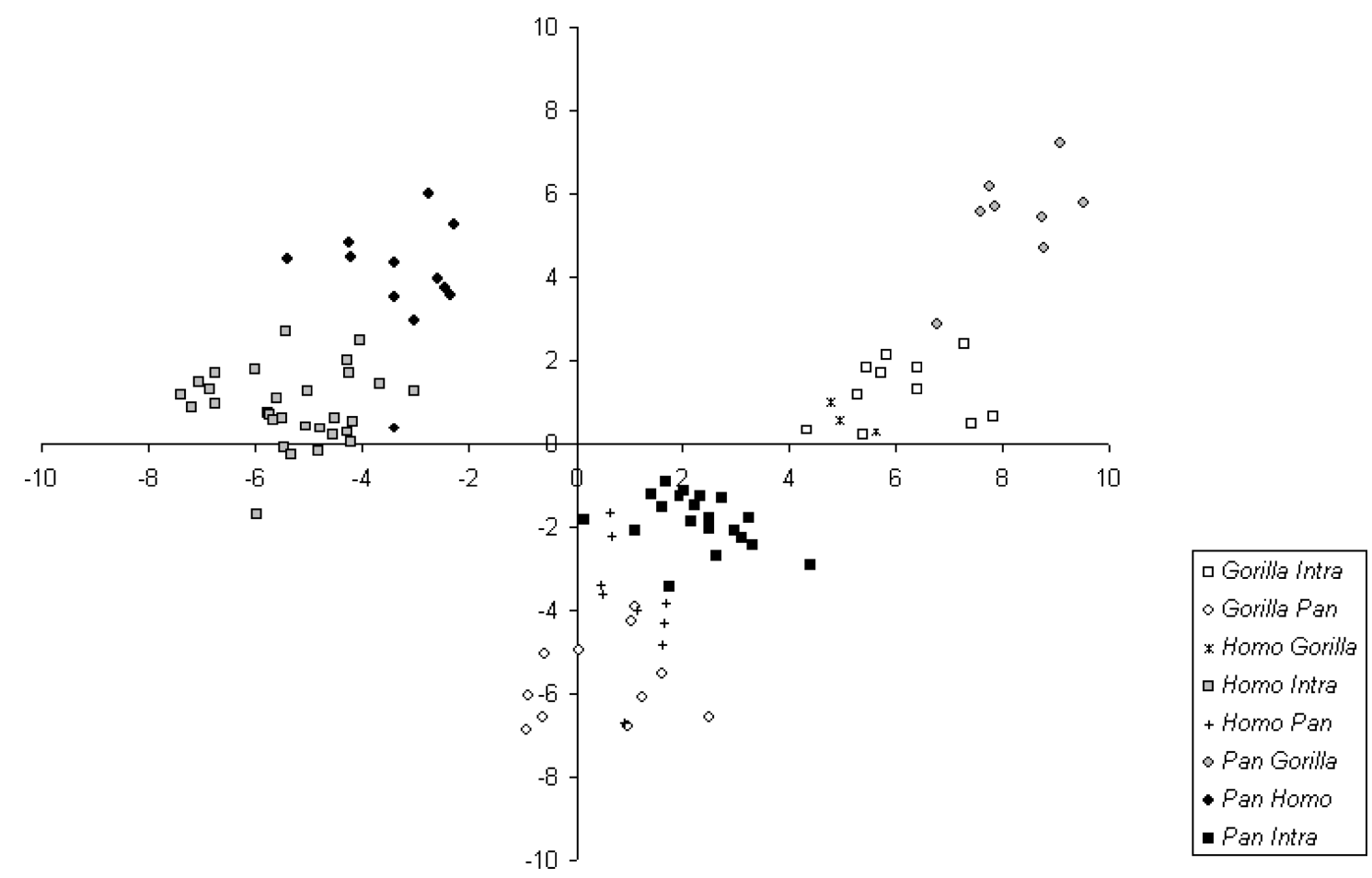

FIGURE 17.5. Scores from discriminant function analysis of the first ten PLS (singular) vectors (after sliding and backscaling) for tibio-talar pairs. DF1 scores on x-axis, DF2 on y-axis. For each interspecific pairing, the first taxon in the legend refers to the tibial dataset, the second to the talar dataset. In the key, "Intra" refers to both matched and mismatched intra-generic pairings.

\subsection{Summary and Conclusions}

2B-PLS analysis of GPA aligned and slid (semi)landmark data from laser scans of Homo, Pan and Gorilla tibiae and tali was undertaken in order to test a new approach for distinguishing between taxa and individuals. When centroid size is restored to the data, the preliminary results of our joint congruence study are encouraging. Thus it appears clear that both size and shape, rather than shape alone, are critical in determining the statistical degree of congruence between the tibial and talar joint surfaces. This may seem intuitive, since when trying to match joints using visual estimation alone, one naturally would discard elements incompatible due to size differences. Shape alone, at least for the surfaces used here, is not a sufficient factor in estimating joint congruence.

In a comparison of all three genera using tibial and talar PLS axis 1 scores, there was reasonably good visual separation $(75 \%$, see Figure 17.3$)$ of intrageneric matches from intergeneric mismatches. Within Homo, individual matches were not distinguished from individual mismatches when the apes were included. However, in a second analysis where apes were not included, $73 \%$ of individual mismatches within Homo were separated from individual matches (Figure 17.4). It is likely that intergeneric differences swamp the subtler distinctions expected within a genus (or species). Therefore, when considering potential hominin fossil material in an analysis such as this one, the important factor is whether the values for that pair of fossils fall far away from the band of matched individuals, or are closer to (or even within) that band.

The DF analysis of the first 10 pairs of singular warp scores yielded good reclassification results, with 101 of 106 pairs correctly identified (over 95\%, as per Table 17.2). Given that DFA considers variation across all variables and compares differences between groups relative to variation within groups, it is not surprising that discrimination is better in Figure 17.5 than in Figure 17.3. However, the basic pattern of group distribution is similar.

This statistical treatment of the PLS scores could therefore be useful for discriminating between matched and unmatched isolated fossil elements (where reciprocal joint surfaces are present). This is particularly true given that the tibial malleolar surface was not included in this analysis, and that the results were nonetheless positive; its inclusion should improve the results. This technique, therefore, has considerable potential for sorting isolated elements recovered from paleontological or archeological sites, in particular, where it is hypothesized that two or more closely related taxa may co-occur.

The results from this study are preliminary, and a number of different directions are currently being explored to further refine our techniques. In particular, we are evaluating different ways of sliding the semilandmarks, so that more 
complex surfaces such as the malleolar facets can be used in future analyses. Further analyses will also benefit from larger sample sizes, which are currently being collected. Most importantly, our next phase of research will incorporate fossil elements into the analysis for the first time.

Acknowledgments. We thank Eric Sargis and Marian Dagosto for inviting us to participate in this volume (and for waiting relatively patiently while we completed our analyses), and Ian Tattersall, Nancy Simmons and Eileen Westwig for access to specimens in their care at the American Museum of Natural History. Thanks also to Ratha Ry, Anthony Pagano, Emily Henderson and Miriam Ruiz for help in laser-scanning and data collection. Comments by two anonymous reviewers and the editors on a previous version helped to improve the manuscript. This research was supported by the National Science Foundation (BCS-0452539). This is NYCEP morphometrics contribution number 18.

\section{References}

Aiello, L. C., Wood B. A., Key, C., Wood, C., 1998. Laser scanning and palaeoanthropology: an example from Olduvai Gorge, Tanzania. In: Strasser, E., Fleagle, J. G., Rosenberger, A. L., McHenry, H. M. (Eds.), Primate Locomotion: Recent Advances. Plenum, New York, pp. 223-236.

Ateshian, G.A., Rosenwasser, M.P., Mow, V.C., 1992. Curvature characteristics and congruence of the thumb carpometacarpal joint: Differences between female and male joints. Journal of Biomechanics 25, 591-607

Bookstein, F. L., 1991. Morphometric Tools for Landmark Data: Geometry and Biology. Cambridge University Press, Cambridge.

Bookstein, F. L., 1997. Landmark methods for forms without landmarks: localizing group differences in outline shape. Medical Image Analysis 1, 225-243.

Bookstein, F. L, Gunz, P., Mitteroecker, P., Prossinger, H., Schaefer, K., Seidler, H., 2003. Cranial integration in Homo: singular warps analysis of the midsagittal plane in ontogeny and evolution. Journal of Human Evolution 44, 167-187.

Bullough, P. G. 1981. The geometry of diarthrodial joints, its physiologic maintenance, and the possible significance of age-related changes in geometry-to-load distribution and the development of osteoarthritis. Clinical Orthopaedics and Related Research 156, 61-66.

Decker, R. L., Szalay, F. S., 1974. Origins and function of the pes in the Eocene Adapidae (Lemuriformes, Primates). In: Jenkins, F. A. (Ed.), Primate Locomotion. Academic, New York, pp. 261-291.

Delson, E., Harvati, K., Reddy, D., Marcus, L. F., Mowbray, K., Sawyer, G. J., Jacob, T., Márquez, S., 2001. The Sambungmacan 3 Homo erectus calvaria: a comparative morphometric and morphological analysis. Anatomical Record 262, 360-377.

Dykyj, D., Ateshian, G. A., Trepal, M. J., MacDonald, L. R., 2001. Articular geometry of the medial tarsometatarsal joint in the foot: comparison of metatarsus primus adductus and metatarsus primus rectus. Journal of Foot and Ankle Surgery 40, 357-365.

Frost, H. M., 1999. Joint anatomy, design, and arthroses: insights of the Utah paradigm. Anatomical Record 255, 162-174.

Frost, S. R., Marcus, L. F., Reddy, D. P., Bookstein, F., Delson, E., 2003. Cranial allometry, phylogeography and systematics of large bodied papionins (Primates: Cercopithecinae) inferred from geometric morphometric analysis of landmark data. Anatomical Record 275A, 1048-1072.

Garcia, G., Harcourt-Smith, W.E.H., 2006. A geometric morphometric analysis of the distal tibia of Homo habilis. American Journal of Physical Anthropology Supplement 42, 92.

Gunz, P., Mitteroecker, P., Bookstein, F. L., 2005. Semilandmarks in three dimensions. In: Slice, D. E. (Ed.), Modern Morphometrics in Physical Anthropology. Kluwer, Dordrecht, The Netherlands, pp. 73-98.

Hamrick, M. W., 1999. A chondral modeling theory revisited. Journal of Theoretical Biology 201, 201-208.

Harcourt-Smith, W.E.H., 2002. Form and Function in the hominoid tarsal skeleton. PhD Thesis. University College London: London.

Harcourt-Smith, W. E. H., Tallman, M., Frost, S. R., Kim, J., Delson, E., 2004. Estimating hominoid reciprocal joint congruence: A comparison of two morphometric techniques. American Journal of Physical Anthropology Supplement 38, 108-109.

Haut, T. L., Hull, M. L., Howell, S. M., 1998. A high-accuracy threedimensional coordinate digitizing system for reconstructing the geometry of diarthrodial joints. Journal of Biomechanics 31, 571-577.

Hertel, R., Lehmann, O., 2001. Glenohumeral joint. Anatomical aspects and implications for prosthesis design. Orthopade 30, 363-369.

Hertel, R., Knothe, U., Ballmer, F. T. 2002. Geometry of the proximal humerus and implications for prosthetic design. Journal of Shoulder and Elbow Surgery 11, 331-338.

Hlavacek, M., Vokoun, D., 1998. The influence of articular surface incongruity on lubrication and contact pressure distribution of loaded synovial joints. Proceedings of the Institute of Mechanical Engineering $[\mathrm{H}]$ 212, 11-22.

Hubberty, C. J., 1994. Applied Discriminant Analysis. Wiley, New York.

Huiskes, R., Kremers, J., de Lange, A., Woltring, H. J., Selvik, G., van Rens, T. J., 1985. Analytical stereophotogrammetric determination of three-dimensional knee-joint geometry. Journal of Biomechanics 18, 559-570.

Kauer, J. M., de Lange, A., 1987. The carpal joint. Anatomy and function. Hand Clinics 3, 23-29.

Kelkar, R., Wang, V. M., Flatow, E. L., Newton, P. M., Ateshian, G. A., Bigliani, L. U., Pawluk, R. J., Mow, V. C., 2001. Glenohumeral mechanics: A study of articular geometry, contact, and kinematics. Journal of Shoulder and Elbow Surgery 10, 73-84.

Lague, M. R., 2003. Patterns of joint size dimorphism in the elbow and knee of catarrhine primates. American Journal of Physical Anthropology 120, 278-297.

Lague, M. R., Jungers, W. L., 1999. Patterns of sexual dimorphism in the hominoid distal humerus. Journal of Human Evolution 36, 379-399.

Leardini, A., 2001. Geometry and mechanics of the human ankle complex and ankle prosthesis design. Clinical Biomechanics 16, 706-709.

Leardini, A., O'Connor, J. J., Catani, F., Giannini, S., 1999. A geometric model of the human ankle joint. Journal of Biomechanics 32, 585-591.

Luckett, W. P., Szalay, F. S. (Eds.), 1975. Phylogeny of the Primates: a Multi-disciplinary Approach. Plenum, New York.

MacLatchy, L. M., 1996. Another look at the australopithecine hip. Journal of Human Evolution 31, 455-476.

MacLatchy, L. M., Bossert, W. H., 1996. An analysis of the articular surface distribution of the femoral head and acetabulum in anthropoids, with implications for hip function in Miocene hominoids. Journal of Human Evolution 31, 425-453. 
Matsuda, S., Matsuda, H., Miyagi, T., Sasaki, K., Iwamoto, Y., Miura, H., 1998. Femoral condyle geometry in the normal and varus knee. Clinical Orthopaedics and Related Research 349, $183-188$.

Medley, J. B., Dowson, D., Wright, V., 1983. Surface geometry of the human ankle joint. Engineering Medicine 12, 35-41.

Mikhail, M.B., Vaswani, A.N., Aloia, J.F., 1996. Racial differences in femoral dimensions and their relation to hip fracture, Osteoporosis International 6(1), 22-24.

O'Higgins, P., 2000. Advances in approaches to the study of morphological variation in the hominid fossil record: Biology, landmarks and geometry. Journal of Anatomy 197, 103-120.

Perez, S. I., Bernal, V., Gonzalez, P. N., 2006. Differences between sliding semilandmark methods in geometric morphometrics: with an application to human craniofacial and dental variation. Journal of Anatomy 208, 769-784.

Pretterklieber, M. L., 1999. Anatomy and kinematics of the human ankle joint. Radiologe 39, 1-7.

Roberts, S. N., Foley, A. P., Swallow, H. M., Wallace, W. A., Coughlan, D. P., 1991. The geometry of the humeral head and the design of prostheses. Journal of Bone and Joint Surgery [Br] 73, 647-650.

Rohlf, F. J., 2005. TpsRelw, v. 1.42. Ecology and Evolution. Stony Brook University, New York.

Rohlf, F. J., Corti, M., 2000. Use of two-block partial leastsquares to study covariation in shape. Systematic Biology 49, $740-753$.

Rohlf, F. J., Slice, D., 1990. Extensions of the Procrustes method for the optimal superimposition of landmarks. Systematic Zoology $39,40-59$.

Rostlund, T., Carlsson, L., Albrektsson, B., Albrektsson, T., 1989. Morphometrical studies of human femoral condyles. Journal of Biomedical Engineering 11, 442-448.

Shiba, R., Sorbie, C., Siu, D. W., Bryant, J. T., Cooke, T. D., Wevers, H. W., 1988. Geometry of the humeroulnar joint. Journal of Orthopaedic Research 6, 897-906.

Siu, D., Rudan, J., Wevers, H. W., Griffiths, P., 1996. Femoral articular shape and geometry. A three-dimensional computerized analysis of the knee. Journal of Arthroplasty 11, 166-173.

Soslowsky, L. J., Flatow, E. L., Bigliani, L. U., Mow, V. C., 1992. Articular geometry of the glenohumeral joint. Clinical Orthopaedics and Related Research 285, 181-190.

Staron, R. B., Feldman, F., Haramati, N., Singson, R. D., Rosenwasser, M., Esser, P. D., 1994. Abnormal geometry of the distal radioulnar joint: MR findings. Skeletal Radiology 23, 369-372.

Staubli, H. U., Durrenmatt, U., Porcellini, B., Rauschning, W., 1999. Anatomy and surface geometry of the patellofemoral joint in the axial plane. Journal of Bone and Joint Surgery [Br] 81, $452-458$.

Swieszkowski, W., Skalski, K., Pomianowski, S., Kedzior, K., 2001. The anatomic features of the radial head and their implication for prosthesis design. Clinical Biomechanics 16, 880-887.

Szalay, F. S., 1968. The beginnings of primates. Evolution 5, 19-36.

Szalay, F. S. (Ed.) 1975 Approaches to Primate Paleobiology. Karger, Basel.

Szalay, F. S., 1977a. Phylogenetic relationships and a classification of the eutherian Mammalia. In: Hecht, M. K., Goody, P. C., Hecht, B. M. (Eds.), Patterns of Vertebrate Evolution. Plenum, New York, pp. 315-374.

Szalay, F. S., 1977b. Ancestors, descendants, sister groups and testing of phylogenetic hypotheses. Systematic Zoology 26, 12-18.
Szalay, F. S., 1984. Arboreality: Is it homologous in metatherian and eutherian mammals? Evolutionary Biology 18, 215-258.

Szalay, F. S., 1993. Species concepts: the tested, the untestable and the redundant. In: Kimbel, W. H., Martin, L. B. (Eds.), Species, Species Concepts, and Primate Evolution. Plenum, New York, pp. 21-42.

Szalay, F. S., 1994. Evolutionary history of the marsupials and an analysis of osteological characters. Cambridge University Press, Cambridge.

Szalay, F. S., 2007. Ancestral locomotor modes, placental mammals, and the origin of Euprimates: Lessons from history. In: Ravosa, M. J., Dagosto, M. (Eds.), Primate Origins: Adaptations and Evolution. Springer, New York, pp. 457-487.

Szalay, F. S., Bock, W. J., 1991. Evolutionary theory and systematics: Relationships between process and patterns. Zeitschrift für zoologische Systematik und Evolutions-Forschung 29, $1-39$.

Szalay, F. S., Dagosto, M., 1980. Locomotor adaptations as reflected on the humerus of Paleogene primates. Folia Primatologica 34, 1-45.

Szalay, F. S., Dagosto, M., 1988. Evolution of hallucial grasping in the primates. Journal of Human Evolution 17, 1-33.

Szalay, F. S., Decker, R. L., 1974. Origins, evolution and function of the tarsus in late Cretaceous eutherians and Paleocene Primates. In: Jenkins, F. A. (Ed.), Primate Locomotion. Academic, New York, pp. 223-259.

Szalay, F. S., Delson, E., 1979. Evolutionary History of the Primates. Academic, New York.

Szalay, F. S., Drawhorn, G., 1980. Evolution and diversification of the Archonta in an arboreal milieu. In: Luckett, W. P. (Ed.), Comparative Biology and Evolutionary Relationships of Tree Shrews. Plenum, New York, pp. 133-169.

Szalay, F. S., Langdon, J., 1986. The foot of Oreopithecus bambolii: An evolutionary assessment. Journal of Human Evolution 15, 585-621.

Szalay, F. S., Lucas, S. G., 1993, Cranioskeletal morphology of archontans, and diagnoses of Chiroptera, Volitantia, and Archonta. In: MacPhee, R. D. E. (Ed.), Primates and their Relatives in Phylogenetic Perspective. Plenum, New York, pp. 187-226.

Szalay, F. S., Lucas, S. G., 1996 The postcranial morphology of Paleocene Chriacus and Mixodectes and the phylogenetic relationships of archontan mammals. Bulletin of New Mexico Museum of Natural History and Science 7, 1-47.

Szalay, F. S., Rosenberger, A. L., Dagosto, M., 1987. Diagnosis and differentiation of the Order Primates. Yearbook of Physical Anthropology 30, 75-105.

Szalay, F. S., Sargis, E. J., 2001. Model-based analysis of postcranial osteology of marsupials from the Paleocene of Itaborai (Brazil) and the phylogenetics and biogeography of Metatheria. Geodiversitas 23, 139-302.

Szalay, F. S., Tattersall, I., Decker, R., 1975. Phylogenetic relationships of Plesiadapis - postcranial evidence. In: Szalay, F. S. (Ed.), Approaches to Primate Paleobiology. Karger, Basel, pp. 136-166.

Tamai, K., Ryu, J., An, K. N., Linscheid, R. L., Cooney, W. P., Chao, E. Y., 1988. Three-dimensional geometric analysis of the metacarpophalangeal joint. Journal of Hand Surgery [Am] 13, 521-529.

Tocheri, M. W., Marzke, M. W., Liu, D., Bae, M., Jones, G. P., Williams, R. C., Razdan, A., 2003. Functional capabilities of modern and fossil hominid hands: Three-dimensional analysis of trapezia. American Journal of Physical Anthropology 122, 101-122.

Trinkaus, E., 2000. Human patellar articular proportions: recent and Pleistocene patterns. Journal of Anatomy 196, 473-483. 
Waide, D. V., Lawlor, G. J., McCormack, B. A., Carr, A. J., 2000. The relationship between surface topography and contact in the elbow joint: development of a two-dimensional geometrical model in the coronal plane. Proceedings of the Institute of Mechanical Engineering [H] 214, 413-423.

Walker, C., Cassar-Pullicino, V. N., Vaisha, R., McCall, I. W., 1993. The patello-femoral joint - a critical appraisal of its geometric assessment utilizing conventional axial radiography and computed arthro-tomography. British Journal of Radiology 66, 755-761.
Wiley, D. F., 2006. Landmark Editor 3.0. Institute for Data Analysis and Visualization, University of California, Davis (http://graphics. idav.ucdavis.edu/research/EvoMorph)

Wood, B. A., Aiello, L. C., Wood, C., Key, C. A., 1998. A technique for establishing the identity of 'isolated' fossil hominid limb bones. Journal of Anatomy 193, 61-72.

Yoshioka, Y., Siu, D. W., Cooke, T. D., Bryant, J. T., Wyss, U., 1988. Geometry of the first metatarsophalangeal joint. Journal of Orthopaedic Research 6, 878-885. 